\title{
TU/e Emonowe

\section{The added value of systematic biopsy in men with suspicion of prostate cancer undergoing multiparametric MRI-targeted biopsy}

Citation for published version (APA):

Mannaerts, C. K., Kajtazovic, A., Lodeizen, O. A. P., Gayet, M., Engelbrecht, M. R. W., Jager, G. J., Wijkstra, H., de Reijke, T. M., \& Beerlage, H. P. (2019). The added value of systematic biopsy in men with suspicion of prostate cancer undergoing multiparametric MRI-targeted biopsy. Urologic oncology, 37(5), 298.e1-298.e9. https://doi.org/10.1016/j.urolonc.2019.01.005

\section{Document license:}

TAVERNE

DOI:

10.1016/j.urolonc.2019.01.005

Document status and date:

Published: 01/05/2019

\section{Document Version:}

Publisher's PDF, also known as Version of Record (includes final page, issue and volume numbers)

\section{Please check the document version of this publication:}

- A submitted manuscript is the version of the article upon submission and before peer-review. There can be important differences between the submitted version and the official published version of record. People interested in the research are advised to contact the author for the final version of the publication, or visit the $\mathrm{DOI}$ to the publisher's website.

- The final author version and the galley proof are versions of the publication after peer review.

- The final published version features the final layout of the paper including the volume, issue and page numbers.

Link to publication

\footnotetext{
General rights

- You may freely distribute the URL identifying the publication in the public portal. follow below link for the End User Agreement:

www.tue.nl/taverne

Take down policy

If you believe that this document breaches copyright please contact us at:

openaccess@tue.nl

providing details and we will investigate your claim.
}

Copyright and moral rights for the publications made accessible in the public portal are retained by the authors and/or other copyright owners and it is a condition of accessing publications that users recognise and abide by the legal requirements associated with these rights.

- Users may download and print one copy of any publication from the public portal for the purpose of private study or research.

- You may not further distribute the material or use it for any profit-making activity or commercial gain

If the publication is distributed under the terms of Article 25fa of the Dutch Copyright Act, indicated by the "Taverne" license above, please 


\title{
The added value of systematic biopsy in men with suspicion of prostate cancer undergoing multiparametric MRI-targeted biopsy
}

\author{
Christophe K. Mannaerts, M.D. ${ }^{a}{ }^{*}$, Amir Kajtazovic, M.D. ${ }^{\text {b }}$, Olivia A.P. Lodeizen, M.D. ${ }^{a}$, \\ Maudy Gayet, M.D. ${ }^{\text {, }}$ Marc R.W. Engelbrecht, Ph.D., M.D. ${ }^{\text {, }}$, Gerrit J. Jager, Ph.D., M.D. e, \\ Hessel Wijkstra, Ph.D., Ir. ${ }^{\text {a,c }}$, Theo M. de Reijke, Ph.D., M.D. ${ }^{a}$, \\ Harrie P. Beerlage, Ph.D., M.D. ${ }^{\text {a,b,c }}$ \\ ${ }^{a}$ Department of Urology, Amsterdam University Medical Centers, University of Amsterdam, Amsterdam, The Netherlands \\ ${ }^{\mathrm{b}}$ Department of Urology, Jeroen Bosch Hospital, 's-Hertogenbosch, The Netherlands \\ ${ }^{\mathrm{c}}$ Department of Electrical Engineering, Eindhoven University of Technology, Eindhoven, The Netherlands \\ ${ }^{\mathrm{d}}$ Department of Radiology, Amsterdam University Medical Centers, University of Amsterdam, Amsterdam, The Netherlands \\ ${ }^{\mathrm{e}}$ Department of Radiology, Jeroen Bosch Hospital, 's-Hertogenbosch, The Netherlands
}

Received 14 September 2018; received in revised form 27 December 2018; accepted 3 January 2019

\begin{abstract}
Purpose: Incorporation of multiparametric magnetic resonance imaging (mpMRI) and targeted biopsy (TBx) in the diagnostic pathway for prostate cancer $(\mathrm{CaP})$ is rapidly becoming common practice. In men with a prebiopsy positive mpMRI a TBx only approach, thereby omitting transrectal ultrasound-guided systematic biopsy ( $\mathrm{SBx}$ ), has been postulated. In this study we evaluated the additional clinical relevance of SBx in men with a positive prebiopsy mpMRI (Prostate Imaging Reporting and Data System [PI-RADS] $\geq 3$ ) undergoing TBx for $\mathrm{CaP}$ detection, Gleason grading and $\mathrm{CaP}$ localization.

Material and methods: Prospective data of 255 consecutive men with a prebiopsy positive mpMRI (PI-RADS $\geq 3$ ) undergoing 12-core SBx and subsequent MRI-transrectal ultrasound fusion TBx in 2 institutions between 2015 and 2018 was obtained. The detection rate for significant $\mathrm{CaP}$ (Gleason score $[\mathrm{GS}] \geq 3+4$ ) for $\mathrm{TBx}$ and $\mathrm{SBx}$ were compared. The rate of potentially missed significant CaP by a TBx only approach was determined and GS concordance and CaP localization by TBx and SBx was evaluated.

Results: TBx yielded significant $\mathrm{CaP}$ in 113 men (44\%) while SBx yielded significant CaP in 110 men $(43 \%)(P=0.856)$. Insignificant $\mathrm{CaP}$ was found in 21 men $(8 \%)$ by TBx, while SBx detected 34 men $(13 \%)$ with insignificant $\mathrm{CaP}(P=0.035)$. A TBx only approach, omitting SBx, would have missed significant $\mathrm{CaP}$ in 13 of the 126 men (10\%) with significant CaP on biopsy. Ten of the 118 men (8\%), both positive on TBx and SBx, were upgraded in GS by SBx while 11 men (9\%) had higher maximum tumor core involvement on SBx. Nineteen of the 97 men (20\%) with significant CaP in both TBx and SBx were diagnosed with unilateral significant CaP on mpMRI and TBx while SBx demonstrated bilateral significant CaP.

Conclusions: In men with a prebiopsy positive mpMRI, TBx detects high-GS CaP while reducing insignificant CaP detection as compared to SBx. SBx and TBx as stand-alone missed significant CaP in $13 \%$ and $10 \%$ of the men with significant CaP on biopsy, respectively. A combination of SBx and TBx remains necessary for the most accurate assessment of detection, grading, tumor core involvement, and localization of CaP. (C) 2019 Elsevier Inc. All rights reserved.
\end{abstract}

Keywords: Prostate cancer; Magnetic resonance imaging; Biopsy; Early diagnosis; Grading

Abbreviations: CI, Confidence interval; DRE, Digital rectal examination; DWI, Diffusion-weighted imaging; DCE, Dynamic contrast-enhanced imaging; GS, Gleason score; (mp)MRI, (multiparametric) magnetic resonance imaging; NPV, negative predictive value; CaP, Prostate Cancer; PSA, Prostate Specific Antigen; RCT, Randomized controlled trial; SBx, Systematic biopsy; T, Tesla; T2W, T2-weighted imaging; TRUS, Transrectal ultrasound; TBx, Targeted fusion biopsy

Funding: This research did not receive any specific grant from funding agencies in the public, commercial, or not-for-profit sectors.
*Corresponding author. Tel.: +31-20-5662979; fax: +31-20-5666030.
E-mail address: c.k.mannaerts @ amc.uva.nl (C.K. Mannaerts). 


\section{Introduction}

Targeted biopsy (TBx) of suspicious lesions on multiparametric magnetic resonance imaging (mpMRI) has demonstrated favorable detection rates of significant prostate cancer $(\mathrm{CaP})$ compared with transrectal ultrasound (TRUS)-guided systematic biopsy ( $\mathrm{SBx}$ ), especially in the repeat-biopsy setting [1,2]. Moreover, TBx of suspicious mpMRI lesions provides for a better Gleason score (GS) prediction of final histopathology compared to SBx. Consequently mpMRI in combination with TBx is increasingly being used [3-5]. Nevertheless, whether SBx can be safely omitted by performing only mpMRI and if necessary TBX is uncertain for now [6-9]. Although this uncertainty primarily focuses on men with a negative mpMRI thereby preventing biopsy at all, information on the necessity of SBx in men with a positive prebiopsy mpMRI undergoing TBX is still undetermined [10].

A TBx only strategy would substantially decrease the number of biopsies, with its associated discomfort, and could reduce detection of insignificant $\mathrm{CaP}$, often associated with overtreatment, as TBx predominantly detects $\mathrm{GS} \geq 7 \mathrm{CaP}$ $[8,11,12]$. Performing SBx, in addition to TBx, however seems to detect some significant $\mathrm{CaP}$ missed on mpMRITBx $[9,13,14]$. Current guidelines on $\mathrm{CaP}$, therefore, still recommend to include systematic biopsies in men with a suspicious mpMRI undergoing TBx $[8,15]$. Proponents of a TBx only pathway, however, emphasize that missed significant tumors on mpMRI are low- to (limited volume) intermediate-risk tumors and are mainly caused by shortcomings in mpMRI and TBx quality. As most of the current studies have poor adherence to the Standards of Reporting for MRI-targeted Biopsy Studies (START) recommendations with widely diverging mpMRI performance, lesion targeting and biopsy procedures, it remains unclear whether SBx could potentially be omitted in men with a positive mpMRI undergoing TBx $[10,16]$. Moreover, the role of SBx for other meaningful factors that influence treatment decisions besides $\mathrm{CaP}$ detection and GS such as tumor focality and cancer core involvement is also unknown. In this study we evaluated the clinical usefulness of SBx, in addition to TBx, in men with a positive prebiopsy mpMRI (PI-RADS $\geq 3$; Prostate Imaging Reporting and Data System v2.0) for CaP detection, Gleason grading and $\mathrm{CaP}$ localization.

\section{Materials and methods}

\subsection{Study population}

From November 2015 to June 2018, a total of 294 biopsy-naïve and 168 prior negative men $(n=462)$ with an elevated prostate-specific antigen (PSA) of $\geq 3.0 \mathrm{ng} / \mathrm{ml}$ and/or abnormal digital rectal examination (DRE), underwent mpMRI of the prostate in 2 institutions, the Amsterdam University Medical Centers, location AMC and Jeroen Bosch Hospital, the Netherlands. MpMRI was generally omitted for men with a PSA level of $>25 \mathrm{ng} / \mathrm{ml}$. Two hundred and sixty four out of 462 (57\%) mpMRIs were classified as suspicious based on a PI-RADS score $\geq 3$ or more. Out of these 264 men, 255 (97\%) underwent both SBx and $\mathrm{TBX}$ and were included in the prospective database approved by the respective institutional review boards and reported according to the START criteria [16].

\section{2. mpMRI protocol}

Of the 255 men, 229 men (90\%) underwent prebiopsy mpMRI at $3.0 \mathrm{~T}$ and $26 \mathrm{men}(10 \%)$ at $1.5 \mathrm{~T}$. One hundred and fourteen mpMRIs (45\%) were performed with T2weighted imaging, diffusion-weighted imaging and dynamic contrast-enhanced imaging (DCE) in 1 center, whereas 141 mpMRIs $(55 \%)$ in the other center were biparametric without DCE imaging. Institutional mpMRI protocols are presented in Appendix 1. At least 1 uroradiologist (M.E., G.J.) with $>8$ years of experience in mpMRI of the prostate analyzed the images. Individual lesions were scored using PI-RADS v2 [17].

\subsection{Biopsy protocols}

Median time between mpMRI and biopsy was 20 days (interquartile range 13-33). SBx and $\mathrm{TBx}$ procedures were performed in 1 session and $\mathrm{SBx}$ was performed before TBx. In 156 out of 255 men (61\%) the SBx operator was unaware of the mpMRI results and TBx procedure planning. Both centers performed a standard 12-core TRUSguided SBx of the peripheral zone with additional cores of the transition zone included in prior-biopsy negative men, if deemed necessary. TBx was performed using MRITRUS fusion techniques: the Artemis system (Eigen, Grass Valley, CA) and the Navigo workstation (UC-Care Medical Systems, Yokneam, Israel) $[18,19]$. Suspicious lesions on mpMRI (PI-RADS $\geq 3$ ) were generally targeted with 2 to 4 cores depending on lesion size. The biopsy procedures were all performed by experienced operators ( $>200$ biopsy cases per year).

\subsection{Histopathology}

Biopsy cores were examined by an uropathologist in each center with $\geq 12$ years of experience. The total number of (positive) cores, the tumor percentage of each biopsy core and GS were reported according to the 2014 International Society of Urological Pathology recommendations [20].

\subsection{Statistical analyses}

Descriptive statistics were used to describe patient characteristics and differences in variables were assessed with the $\chi^{2}$ test for categorical variables and Mann-Whitney $U$ test for continuous variables. Clinically significant $\mathrm{CaP}$ was 
defined as GS $\geq 3+4=7$ in a biopsy core. Detection rates were presented using cross-tabulations and compared using the McNemar test. We determined the number of missed and GS undergraded clinically significant $\mathrm{CaP}$ on TBx and evaluated the role of mpMRI reading and biopsy lesion targeting in men with missed significant $\mathrm{CaP}$ on $\mathrm{TBx}$ using mpMRI information and positive SBx locations. Last, we compared positive (significant) SBx and TBx core locations within each prostate lobe to assess the role of TBx and SBx in multifocality of $\mathrm{CaP}$ disease.

\section{Results}

\subsection{Patient and procedure characteristics}

Median age in the cohort of 255 men was 65 years (interquartile range 61-69) as seen in Table 1. Out of the 255 men, 160 (63\%) had a CaP positive biopsy. Men with a positive biopsy were significantly older (66 vs. 64 years; $P=0.04)$, biopsy-naïve (78\% vs. $38 \%$ ) and had more often an abnormal DRE (53\% vs. 7\%; $P<0.001)$ compared with men with a negative biopsy. Men with a positive biopsy had a higher overall PI-RADS score $(P<0.001)$ while the amount of cores taken per procedure were comparable between both groups with a median of $3 \mathrm{TBx}$ cores per procedure.

\subsection{Detection of $\mathrm{CaP}$}

Of the 160 men with a positive biopsy, $126(79 \%)$ were diagnosed with significant $\mathrm{CaP}(\mathrm{GS} \geq 3+4)$ and $34(21 \%)$ with insignificant $\mathrm{CaP}$ (GS $3+3$ ) as seen in Table 2. TBx revealed significant $\mathrm{CaP}$ in 113 men $(44 \%)$ while $\mathrm{SBx}$ revealed significant $\mathrm{CaP}$ in 110 men (43\%; $P=0.856)$. Insignificant $\mathrm{CaP}$ was found in 21 men (8\%) by $\mathrm{TBx}$ and in 34 men by $\mathrm{SBx}(13 \% ; P=0.035)$. GS $\geq 4+3 \mathrm{CaP}$ was found via TBx in 69 men (27\%) compared to 56 men $(22 \%)$ via SBx $(P=0.019)$. In men with PI-RADS 3, TBx detected 14 out of the 20 men (70\%) with significant CaP on biopsy. For PI-RADS 4 and 5, 48 men and 58 men had significant $\mathrm{CaP}$ on biopsy with $\mathrm{TBx}$, detecting significant $\mathrm{CaP}$ in 43 men (90\%) and 56 men (97\%), respectively. Detection results for TBx and SBx is presented separately for biopsy-naïve men and prior-negative men in Appendix 2.

\subsection{Missed and GS undergraded significant $\mathrm{CaP}$}

TBx detected 16 out of 126 men (13\%) with significant $\mathrm{CaP}(5 \mathrm{GS} 3+4=7,5 \mathrm{GS} 4+3=7$ and $6 \mathrm{GS} \geq 4+4=8$ ) that were missed $(n=10)$ or GS undergraded insignificant $(n=6)$ on SBx as seen in Table 3. TBx would have omitted detection of insignificant $\mathrm{CaP}$ in 13 of the 34 men (38\%). $\mathrm{SBx}$ detected 13 out of 126 men (10\%) with significant $\mathrm{CaP}$ ( 9 GS $3+4=7,3$ GS $4+3=7$ and $1 \mathrm{GS} \geq 4+4=8$ ) that were missed $(7 \%, n=9)$ or GS undergraded insignificant $(3 \%, n=4)$ on TBx. Significant $\mathrm{CaP}$ on $\mathrm{TBx}$ was missed in
4 men with PI-RADS 3, 4 men with PI-RADS 4 and 1 man with PI-RADS 5, respectively. Data of these men is descriptively summarized in Appendix 3. Two men with PI-RADS 3 and 1 man for both PI-RADS 4 and 5 were diagnosed as GS $3+3=6$ on TBx while SBx demonstrated significant $\mathrm{CaP}$. Data of these men is descriptively summarized in Appendix 4.

\subsection{GS concordance and maximum tumor core involvement}

Of the 118 men both positive on SBx and TBx, 91 men (77\%) had concordant GS, while 17 (14\%) and 10 men (8\%) were GS upgraded on TBx and SBx, respectively (Table 4). Of the 10 men upgraded on SBx, 4 men (3\%) were upgraded from insignificant to significant $\mathrm{CaP}$. One hundred and seven men $(91 \%)$ had equal $(n=91)$ or higher $(n=16)$ maximum tumor core involvement in TBx compared to SBx. Eleven men (9\%) had higher maximum tumor core involvement on SBx.

\subsection{Focality of disease}

As shown in Table 5, 75 out of the 118 men (64\%) both positive on $\mathrm{SBx}$ and $\mathrm{TBx}$, had $\mathrm{CaP}$ detected with concordant unilateral or bilateral disease on SBx and TBx. For significant $\mathrm{CaP}$ only, 72 out of the 97 men $(74 \%)$ had significant $\mathrm{CaP}$ detected with concordant unilateral or bilateral disease as defined on SBX and TBX while 21 of the 97 men $(22 \%)$ were diagnosed with unilateral significant $\mathrm{CaP}$ on TBx with $\mathrm{SBx}$ demonstrating significant $\mathrm{CaP}$ on the contralateral side $(n=2)$ or bilateral in the prostate $(n=19)$.

\section{Discussion}

SBx in men with an elevated PSA level and/or abnormal DRE has been the cornerstone of CaP diagnosis for decades. Due to its ability to detect significant $\mathrm{CaP}$ while reducing overdetection of indolent disease, mpMRI and TBx are rapidly becoming common practice in the diagnostic pathway and questions arise whether SBx can be fully replaced by this novel strategy $[2,5,21,22]$. Results of our study in 255 men with a prebiopsy positive mpMRI undergoing both SBx and TBx demonstrated that a TBx only approach would have missed significant $\mathrm{CaP}$ in 9 men (7\%) and misclassified $\mathrm{CaP}$ as insignificant in 4 more men (3\%). Using mpMRI information and positive SBx core locations, both inadequate $\mathrm{CaP}$ visualization on mpMRI $(n=8)$ and erroneous biopsy lesion targeting $(n=5)$ contributed to missed significant $\mathrm{CaP}$ in a mpMRI-TBx pathway. In addition, additional $\mathrm{SBx}$ for detection of significant $\mathrm{CaP}$ seems more useful in men with an intermediate suspicion on mpMRI as TBx detected $97 \%(56 / 58)$ of the significant $\mathrm{CaP}$ in men with a PI-RADS score 5 compared to $70 \%(14 / 20)$ in men with a PI-RADS score 3. For detection of significant $\mathrm{CaP}$, one might consider to omit SBx in men with PI-RADS 
Table 1

Clinical characteristics.

\begin{tabular}{|c|c|c|c|c|}
\hline Variable & Total $(N=255)$ & Positive biopsy $(N=160)$ & Negative biopsy $(N=95)$ & $P$ value \\
\hline \multicolumn{5}{|l|}{ Patient } \\
\hline Age at biopsy; y, median (IQR) & $65(61-69)$ & $66(62-71)$ & $64(59-68)$ & 0.044 \\
\hline \multicolumn{5}{|l|}{ Ethnicity, $n(\%)$} \\
\hline - Caucasian & $233(91)$ & $141(88)$ & $92(97)$ & $\mathbf{0 . 0 1 7}$ \\
\hline - Non-Caucasian & $22(9)$ & $19(12)$ & $3(3)$ & \\
\hline Prebiopsy PSA; ng/ml, median (IQR) & $8.1(5.9-12.0)$ & $8.1(5.9-12.0)$ & $8.3(5.9-12.0)$ & 0.781 \\
\hline PSA density; ng/ml/ml, median (IQR) & $0.18(0.12-0.28)$ & $0.21(0.13-0.30)$ & $0.24(0.20-0.23)$ & $<0.001$ \\
\hline \multicolumn{5}{|l|}{ DRE, $n(\%)$} \\
\hline$\bullet$ Normal & $163(64)$ & $75(47)$ & $88(93)$ & $<0.001$ \\
\hline - Abnormal & $92(36)$ & $85(53)$ & $7(7)$ & \\
\hline TRUS prostate volume; cc, median (IQR) & $45(34-63)$ & $40(30-53)$ & $54(42-72)$ & $<0.001$ \\
\hline \multicolumn{5}{|l|}{ Biopsy type, $n(\%)$} \\
\hline$\bullet$ Biopsy-naïve & $161(63)$ & $125(78)$ & $36(38)$ & $<0.001$ \\
\hline$\bullet$ Prior negative & $94(37)$ & $35(22)$ & $59(62)$ & \\
\hline \multicolumn{5}{|l|}{ Biopsy session, $n(\%)$} \\
\hline$\bullet 1$ & $161(63)$ & $125(78)$ & $36(38)$ & $<0.001$ \\
\hline • 2 & $56(22)$ & 27 (17) & $29(31)$ & \\
\hline$\bullet \geq 3$ & $38(15)$ & $8(5)$ & $30(31)$ & \\
\hline \multicolumn{5}{|l|}{ mpMRI } \\
\hline \multicolumn{5}{|l|}{ PI-RADS score per patient, $n(\%)^{\mathrm{a}}$} \\
\hline$\bullet 3$ & $81(32)$ & $28(18)$ & $53(56)$ & $<0.001$ \\
\hline$\bullet 4$ & $94(37)$ & $63(39)$ & $31(33)$ & \\
\hline$\bullet 5$ & $80(31)$ & $69(43)$ & $11(12)$ & \\
\hline \multicolumn{5}{|l|}{ MRI lesions per patient, $n(\%)$} \\
\hline$\bullet 1$ & $186(73)$ & $114(71)$ & $72(76)$ & 0.742 \\
\hline$\bullet 2$ & $62(24)$ & $41(26)$ & $21(22)$ & \\
\hline$\bullet \geq 3$ & 7 (3) & $5(3)$ & $2(2)$ & \\
\hline \multicolumn{5}{|l|}{ Biopsy } \\
\hline \multicolumn{5}{|l|}{ SBx cores in biopsy-naïve patients, $\mathrm{n}(\%)$ : } \\
\hline$\bullet<12$ & $8(5)$ & $7(6)$ & $1(3)$ & 0.649 \\
\hline$\bullet 12$ & $147(91)$ & $114(91)$ & $33(92)$ & \\
\hline$\bullet>12$ & $6(4)$ & $4(3)$ & $2(6)$ & \\
\hline \multicolumn{5}{|l|}{ SBx cores in prior-negative patients, $n(\%)$} \\
\hline$\bullet<12$ & $9(9)$ & $3(9)$ & $6(10)$ & 0.596 \\
\hline$\bullet 12-16$ & $56(60)$ & $17(54)$ & $37(63)$ & \\
\hline$\bullet 16$ & $29(31)$ & $13(37)$ & $16(27)$ & \\
\hline MRI-TBx cores patient, median (range) & $3(2-4)$ & $3(2-4)$ & $3(2-4)$ & 0.131 \\
\hline \multicolumn{5}{|l|}{ MRI-TBx cores per lesion, $n(\%)$} \\
\hline$\bullet 1, n(\%)$ & $22(7)$ & $13(6)$ & $9(7)$ & 0.279 \\
\hline$\bullet 2, n(\%)$ & $220(67)$ & $136(65)$ & $84(70)$ & \\
\hline$\bullet 3, n(\%)$ & $61(18)$ & $45(21)$ & $16(13)$ & \\
\hline$\bullet 4, n(\%)$ & $21(6)$ & $12(6)$ & $9(7)$ & \\
\hline$\bullet \geq 5, n(\%)$ & $7(2)$ & $5(2)$ & $2(2)$ & \\
\hline \multicolumn{5}{|l|}{ Biopsy complication: } \\
\hline Yes & & & & 0.574 \\
\hline - Prostatitis, $n(\%)$ & $10(4)$ & $6(4)$ & $4(4)$ & \\
\hline - Urinary retention, $n(\%)$ & $1(0.4)$ & $1(0.5)$ & - & \\
\hline - Gross rectal bleeding, $n(\%)$ & $1(0.4)$ & - & $1(1)$ & \\
\hline • Gross hematuria & $1(0.4)$ & $1(0.5)$ & - & \\
\hline No, $n(\%)$ & $242(95)$ & $152(95)$ & $90(95)$ & \\
\hline
\end{tabular}

$\mathrm{DRE}=$ digital rectal examination; IQR = interquartile range; PI-RADS = Prostate Imaging Reporting and Data System; PSA = prostate specific antigen; $\mathrm{SBx}=$ systematic biopsy; TBx = targeted biopsy; TRUS $=$ transrectal ultrasound.

${ }^{\mathrm{a}}$ The highest PI-RADS score is given if more than one lesion was present in a patient.

Statistically significant $\mathrm{p}$-values $(\mathrm{p}<0.05)$ are indicated in bold. 
Table 2

Prostate cancer detection outcomes stratified according to the PI-RADS score.

\begin{tabular}{|c|c|c|c|c|}
\hline Detection & Men with PI-RADS $3(n=81)$ & Men with PI-RADS $4(n=94)$ & Men with PI-RADS $5(n=80)$ & Total $(n=255)$ \\
\hline \multicolumn{5}{|l|}{ All biopsy (TBx and SBx) } \\
\hline No $\mathrm{CaP}, n(\%)$ & $53(65)$ & $31(33)$ & $11(14)$ & $95(37)$ \\
\hline \multicolumn{5}{|l|}{ Insignificant CaP, $n(\%)$} \\
\hline - GS $3+3$ & $8(10)$ & $15(16)$ & $11(14)$ & $34(13)$ \\
\hline \multicolumn{5}{|l|}{ Significant CaP, $n(\%)$} \\
\hline - All & $20(25)$ & $48(51)$ & $58(72)$ & $126(49)$ \\
\hline o GS $3+4$ & $12(15)$ & $15(16)$ & $23(29)$ & $50(20)$ \\
\hline o GS $4+3$ & $7(9)$ & $24(25)$ & $12(15)$ & $43(17)$ \\
\hline o $\mathrm{GS} \geq 4+4$ & $1(1)$ & $9(10)$ & $23(29)$ & $33(13)$ \\
\hline Total & $81(100)$ & $94(100)$ & $80(100)$ & 255 \\
\hline \multicolumn{5}{|l|}{ TBx only } \\
\hline No CaP, $n(\%)$ & $61(75)$ & $44(47)$ & $16(20)$ & $121(47)$ \\
\hline o with significant $\mathrm{CaP}$ on $\mathrm{SBx}$ & $4(5)$ & $4(4)$ & $1(1)$ & $9(4)$ \\
\hline \multicolumn{5}{|l|}{ Insignificant $\mathrm{CaP}, \mathrm{n}(\%)$} \\
\hline$\bullet \mathrm{GS} 3+3$ & $6(7)$ & $7(7)$ & $8(10)$ & $21(8)$ \\
\hline o with significant $\mathrm{CaP}$ on $\mathrm{SBx}$ & $2(2)$ & $1(1)$ & $1(1)$ & $4(2)$ \\
\hline \multicolumn{5}{|l|}{ Significant $\mathrm{CaP}, n(\%)$} \\
\hline - All: & $14(17)$ & $43(46)$ & $56(70)$ & $113(44)$ \\
\hline o GS $3+4$ & $7(9)$ & $15(16)$ & $22(28)$ & $44(17)$ \\
\hline o GS $4+3$ & $7(9)$ & $20(21)$ & $13(16)$ & $40(16)$ \\
\hline o GS $\geq 4+4$ & 0 & $8(9)$ & $21(26)$ & $29(11)$ \\
\hline Total, $n(\%)$ & $81(100)$ & $94(100)$ & $80(100)$ & $255(100)$ \\
\hline \multicolumn{5}{|l|}{ SBx only } \\
\hline No $\mathrm{CaP}, n(\%)$ & $56(69)$ & $35(37)$ & $20(25)$ & $111(44)$ \\
\hline $\mathrm{o}$ with significant $\mathrm{CaP}$ on $\mathrm{TBx}$ & $1(1)$ & $2(2)$ & $7(9)$ & $10(4)$ \\
\hline \multicolumn{5}{|l|}{ Insignificant $\mathrm{CaP}, n(\%)$} \\
\hline - GS $3+3$ & $7(9)$ & $17(18)$ & $10(13)$ & $34(13)$ \\
\hline o with significant $\mathrm{CaP}$ on $\mathrm{TBx}$ & $1(1)$ & $4(4)$ & $1(1)$ & $6(2)$ \\
\hline \multicolumn{5}{|l|}{ Significant CaP, $n(\%)$} \\
\hline - All & $18(22)$ & $42(45)$ & $50(63)$ & $110(44)$ \\
\hline o GS $3+4$ & $12(15)$ & $20(21)$ & $22(28)$ & $54(21)$ \\
\hline o GS $4+3$ & $5(6)$ & $16(17)$ & $10(13)$ & $31(12)$ \\
\hline o $\mathrm{GS} \geq 4+4$ & $1(1)$ & $6(6)$ & $18(23)$ & $25(10)$ \\
\hline Total, $n(\%)$ & $81(100)$ & $94(100)$ & $80(100)$ & $255(100)$ \\
\hline
\end{tabular}

GS = Gleason score; MRI = magnetic resonance imaging; $\mathrm{CaP}=$ prostate cancer; PI-RADS $=$ Prostate Imaging Reporting and Data System; SBx = systematic biopsy; TBx = targeted biopsy.

score 5 as additional benefit of SBx is very low compared to the high amount of biopsies that need to be taken.

Our results are in line with a recent literature review that demonstrated that combination of both $\mathrm{TBx}$ and $\mathrm{SBx}$ improved significant $\mathrm{CaP}$ detection rates with $5 \%$ to $15 \%$ as compared with TBx alone [10]. Our study adds to this review other new important findings. First of all, maximum tumor core involvement is underestimated by $\mathrm{TBx}$ in $9 \%$ (11/118) of the men both positive on SBx and TBx while 5 more men (4\%) had a higher significant GS in the SBx compared to the TBx. Although one could argue that TBx precision was insufficient in these cases, $\mathrm{TBx}$ fusion cores are also known for their 2 to $3 \mathrm{~mm}$ error margin while a recent systematic review did not demonstrate any additional value of in-bore MRI TBx compared to MRI-TRUS fusion TBx [23-25]. Moreover, 21 of the 97 men (22\%) both positive for significant $\mathrm{CaP}$ on $\mathrm{TBx}$ and $\mathrm{SBx}$ were diagnosed with unilateral disease on mpMRI-TBx while SBx demonstrated bilateral significant disease. In line with the literature, TBx has a high sensitivity for index lesion characterization, but secondary lesions are often missed by imaging while disease progression is not always driven by the index lesion only [22,26-29]. Notwithstanding the fact that $\mathrm{CaP}$ detection and accurate GS determination are the cornerstone for decision making in $\mathrm{CaP}$, secondary pathological features such as maximum tumor core involvement and multifocality are relevant for adequate risk stratification, prognosis, and treatment evaluation of $\mathrm{CaP}$. Especially, for novel focal therapy techniques, aiming at selectively ablating CaP tumors while sparing functional and anatomical structures, mpMRI and TBx should be combined with SBx for adequate patient selection [30].

Despite the drawbacks of performing only TBx, the TBx approach was more efficient on a per-core basis in the detection of significant $\mathrm{CaP}$ with higher rates of positive cores and 4 times fewer biopsy cores (median cores per session: 12 vs. 
Table 3

Cross tabulation of the MRI-TBx and SBx protocol for detection and Gleason score.

Insignificant/Significant $(n=255)$

MRI TBx protocol, $n(\%)$

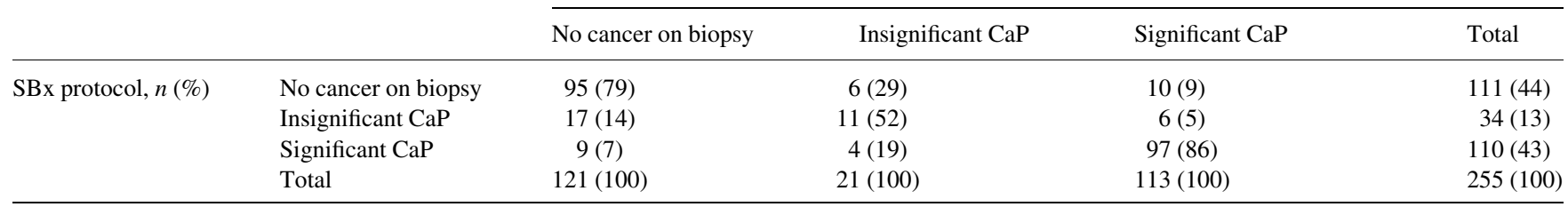

Gleason Score (GS) $(n=255)$

\begin{tabular}{|c|c|c|c|c|c|c|c|}
\hline & & \multicolumn{6}{|c|}{ MRI TBx protocol, $n(\%)$} \\
\hline & & No cancer on biopsy & GS $3+3$ & $\mathrm{GS} 3+4$ & $\mathrm{GS} 4+3$ & $\mathrm{GS} \geq 4+4$ & Total \\
\hline \multirow[t]{6}{*}{$\mathrm{SBx}$ protocol, $n(\%)$} & No cancer on biopsy & $95(78)$ & $6(29)$ & $3(6)$ & $3(8)$ & $4(14)$ & $111(44)$ \\
\hline & GS $3+3$ & $17(14)$ & $11(52)$ & $2(4)$ & $3(8)$ & $1(3)$ & 34 (13) \\
\hline & GS $3+4$ & $7(6)$ & $2(10)$ & $36(77)$ & $8(22)$ & $1(3)$ & $54(21)$ \\
\hline & GS $4+3$ & $2(2)$ & $1(5)$ & $3(7)$ & $23(59)$ & $2(7)$ & $32(13)$ \\
\hline & $\mathrm{GS} \geq 4+4$ & 0 & $1(5)$ & 0 & $3(8)$ & $21(72)$ & $24(9)$ \\
\hline & Total & $121(100)$ & $21(100)$ & $44(100)$ & $40(100)$ & $29(100)$ & $255(100)$ \\
\hline
\end{tabular}

$\mathrm{GS}=$ Gleason score $\mathrm{MRI}=$ magnetic resonance imaging $; \mathrm{CaP}=$ prostate cancer; $\mathrm{SBx}=$ systematic biopsy $; \mathrm{TB}=$ targeted biopsy .

Table 4

GS concordance and biopsy core outcomes stratified according to the PI-RADS score.

\begin{tabular}{|c|c|c|c|c|}
\hline & Men with PI-RADS $3(n=81)$ & Men with PI-RADS $4(n=94)$ & Men with PI-RADS $5(n=80)$ & Total $(n=255)$ \\
\hline \multicolumn{5}{|c|}{ Gleason score (GS) concordance in men with TBx and SBx both CaP-positive $(n=118)$} \\
\hline Concordant, $n(\%)$ & $12(71)$ & $31(67)$ & $48(87)$ & $91(77)$ \\
\hline Upgrading on TBx, $n(\%)$ : & $1(6)$ & $12(26)$ & $4(7)$ & $17(14)$ \\
\hline Upgrading on SBx, $n(\%)$ : & $4(23)$ & $3(7)$ & $3(5)$ & $10(8)$ \\
\hline Total, $n(\%)$ & $17(100)$ & $46(100)$ & $55(100)$ & $118(100)$ \\
\hline \multicolumn{5}{|c|}{ MTCI in men with TBx and SBx both CaP-positive $(n=118)$} \\
\hline Equal MTCI in $\%, n(\%)$ & $13(76)$ & $33(72)$ & $45(82)$ & $91(77)$ \\
\hline Higher MTCI in $\%$ on TBx, $n(\%)$ & $1(6)$ & $8(17)$ & $7(13)$ & $16(14)$ \\
\hline Higher MTCI in \% on SBx, $n(\%)$ & $3(18)$ & $5(11)$ & $3(5)$ & $11(10)$ \\
\hline Total, $n(\%)$ & $17(100)$ & $46(100)$ & $55(100)$ & $118(100)$ \\
\hline \multicolumn{5}{|l|}{ Positive biopsy cores: TBx only } \\
\hline \multicolumn{5}{|l|}{ Positive CaP cores, $n(\%)$ : } \\
\hline$\bullet \mathrm{GS} 6 \mathrm{CaP}$ & $10(4)$ & $16(5)$ & $20(8)$ & $46(6)$ \\
\hline$\bullet \mathrm{GS} \geq 7 \mathrm{CaP}$ & $28(13)$ & $90(31)$ & $138(55)$ & $256(33)$ \\
\hline Total cores $(n)$ & $223(100)$ & $294(100)$ & $253(100)$ & $770(100)$ \\
\hline \multicolumn{5}{|l|}{ Positive biopsy cores: SBx only } \\
\hline \multicolumn{5}{|l|}{ Positive CaP cores, $n(\%)$ : } \\
\hline$\bullet \mathrm{GS} 6 \mathrm{CaP}$ & $38(4)$ & $63(5)$ & $74(8)$ & $175(6)$ \\
\hline - $\mathrm{GS} \geq 7 \mathrm{CaP}$ & $52(5)$ & $191(16)$ & $281(30)$ & $524(17)$ \\
\hline Total cores, $n(\%)$ & $1021(100)$ & $1178(100)$ & $940(100)$ & $3139(100)$ \\
\hline \multicolumn{5}{|c|}{ Number of cores per significant CaP diagnosis, mean [median] (IQR) } \\
\hline $\operatorname{TBx}(n=113)$ & $2.8[3](2-3)$ & $3.1[3](2-4)$ & $3.2[3](2-4)$ & $3.0[3](2-4)$ \\
\hline $\operatorname{SBx}(n=110)$ & $12.6[12](12-12)$ & $12.5[12](12-12)$ & $11.8[12](12-12)$ & $12.3[12](12-12)$ \\
\hline
\end{tabular}

$\mathrm{GS}=$ Gleason score $\mathrm{MTCI}=$ maximum tumor core involvement, $\mathrm{CaP}=$ prostate cancer; $\mathrm{PI}-\mathrm{RADS}=$ Prostate imaging reporting and data system; $\mathrm{SBx}=$ systematic biopsy; TBx $=$ targeted biopsy.

3) per diagnosis. Moreover, a SBx only approach would have also missed a non-negligible amount (13\%) of significant $\mathrm{CaP}$ while performing additional SBx on top of TBx, as expected, will come with an increased detection of insignificant $\mathrm{CaP}$ as a downside. This is demonstrated by the $38 \%$ additional insignificant $\mathrm{CaP}$ found by $\mathrm{SBx}$ alone in our cohort.

While reduced detection of insignificant $\mathrm{CaP}$ by $\mathrm{TBx}$ is demonstrated in almost all recent literature, results on 
Table 5

Cross tabulation of the MRI-TBx and SBx protocol for (significant) CaP tumor localization.

Unilateral/bilateral disease (all CaP) $(n=160)$

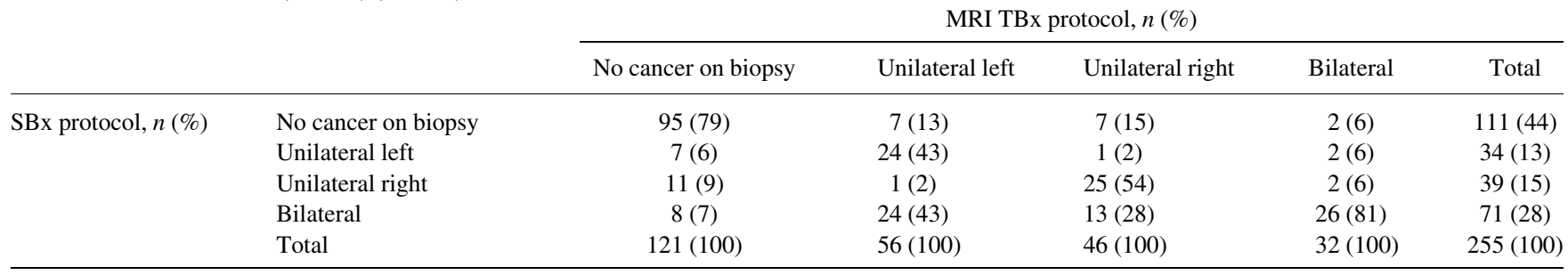

Unilateral/bilateral disease (significant $\mathrm{CaP})(n=160)$

MRI TBx protocol, $n(\%)$

\begin{tabular}{|c|c|c|c|c|c|c|}
\hline & & \\
\hline & & No cancer/insignificant & Unilateral left & Unilateral right & Bilateral & Total \\
\hline \multirow[t]{5}{*}{ SBx protocol, $n(\%)$} & No cancer /insignificant & $129(91)$ & $7(14)$ & $6(16)$ & $3(12)$ & $145(57)$ \\
\hline & Unilateral left & $5(4)$ & $29(58)$ & $1(3)$ & $3(12)$ & $38(15)$ \\
\hline & Unilateral right & $7(5)$ & $2(4)$ & $24(63)$ & 0 & $33(13)$ \\
\hline & Bilateral & $1(1)$ & $12(24)$ & $7(18)$ & $19(76)$ & $39(15)$ \\
\hline & Total & $142(100)$ & $50(100)$ & $38(100)$ & $25(100)$ & $255(100)$ \\
\hline
\end{tabular}

$\mathrm{MRI}=$ magnetic resonance imaging; $\mathrm{CaP}=$ prostate cancer $\mathrm{SBx}=$ systematic biopsy; $\mathrm{TBx}=$ targeted biopsy.

increased detection of significant $\mathrm{CaP}$ by TBx are mixed $[1,31]$. In our study, high GS CaP (GS $\geq 4+3)$ was more often found by TBx compared to $\mathrm{SBx}(27 \%$ vs. $22 \%$; $P=$ 0.035 ), but both techniques detected an equal amount of GS $\geq 3+4 \mathrm{CaP}$ ( $44 \%$ vs. $43 \% ; P=0.856$ ). Two recently published prospective, multicenter, paired, diagnostic studies in biopsy-naïve men (MRI-FIRST and 4M study) demonstrated comparable detection of significant $\mathrm{CaP}$ for $\mathrm{SBx}$ and TBx while the multicenter PRECISION randomized controlled trial demonstrated that mpMRI with $\mathrm{TBx}$ detected more significant $\mathrm{CaP}$ compared to $\mathrm{SBx}[2,32,33]$. Despite the differences in study design, comparison of results with these studies with fairly similar baseline characteristics illustrated some important findings (Appendix 5). Detection rates of significant $\mathrm{CaP}$ by TBx were higher in the PRECISION trial than in the biopsy-naive men of the other studies: $38 \%$ vs. $32 \%$ (current study), $32 \%$ (MRIFIRST) and 25\% (4M study), respectively. However, significant $\mathrm{CaP}$ on $\mathrm{SBx}$ was detected in $35 \%$ and $30 \%$ of all biopsy-naïve men in our study and the MRI-FIRST study, while the PRECISION randomized controlled trial and $4 \mathrm{M}$ study detected only $26 \%$ and $23 \%$ of the men with significant $\mathrm{CaP}$ on SBx, respectively. Despite the clear difference in level of evidence, comparisons like these demonstrate that there is still room for improvement in attaining consistency in not only mpMRI with TBx but also in SBx since much higher detection rates of significant $\mathrm{CaP}$ were achieved with SBx in our study. Especially given the fact that biopsy sessions in biopsy-naïve men were performed by 2 operators in a complete blinded fashion in $86 \%$ (252/294) of the cases, mpMRI reading could have positively influenced the SBx performance only in a minority of the cases. Moreover, sensitivity analysis excluding these cases showed no impact on the detection rate of SBx indicating that these results most likely reflect the true clinical performance of SBx when performed by experienced TRUS operators.

Besides this limitation our study has other limitations. One being the fact that our study does not address the full diagnostic pathway of mpMRI and TBx, but only the clinical relevance of SBx in men with a positive prebiopsy mpMRI undergoing TBx. Second, SBx harbors both random and systematic errors as shown in studies as the PROMIS trial with transperineal template mapping biopsy as reference standard and studies in the repeat biopsy setting showing non-negligible rates of significant $\mathrm{CaP}$ after a negative SBx.[6,34,35] Consequently, the use of SBx as reference standard comes with limitations. Comparison with radical prostatectomy pathology, however, was also not possible, as only a selected group underwent surgery and partial embedding was performed in the majority of these patients. Furthermore, our study addressed the more clinical question as to whether TBx is adequate as a standalone approach for $\mathrm{CaP}$ detection and localization in mpMRI positive men as in practice men with a positive mpMRI result could then avoid the need for SBx. Third, SBx was performed before TBx to evaluate the unbiased performance of SBx. This may have resulted in nonuniform prostate swelling making image registration and TBx less accurate. The diagnostic yield of TBx in men with a positive prebiopsy mpMRI, however, was on average comparable with current literature as shown in Appendix 5. Fourth, 1 center did not perform DCE MRI while 10\% of all mpMRI scans were performed on $1.5 \mathrm{~T}$. Both could have negatively affected TBx outcome. Recent studies, however, demonstrated comparable diagnostic accuracy of PI-RADS v2 using a biparametric protocol while mpMRI on $1.5 \mathrm{~T}$ is capable of yielding adequate diagnostic scans [17,36,37]. Lastly, our chosen definition of clinically significant $\mathrm{CaP}$ is 
debatable. Although it is common to define GS $\geq 3+4$ disease on biopsy as significant $\mathrm{CaP}$, GS $3+4=7$ disease shows considerable heterogeneity in pathological features and clinical outcome [38]. In the future, addition of histopathological parameters such as cribriform growth pattern and percentage Gleason grade 4 could improve risk stratification of GS $3+4$ disease and possibly aid in reaching a more definitive answer to the question whether TBx could safely replace SBx as the majority (69\%) of missed tumors with a TBx only pathway were tumors with a GS $3+4=7$.

Findings of this study highlight that for now, both TBx and SBx are important in the detection, grading and localization of CaP. Due to the major implications for broader use of mpMRI such as additional equipment, trained personnel, and total costs, findings like these should be extended. There is a well-known variation in biopsy targeting and a substantial portion of false positives is reported in literature [10]. Comparison of detection rates between our 2 centers also demonstrated significantly different detection rates for $\mathrm{CaP}$ on $\mathrm{TBx}(76 \%$ vs. $41 \%, P<0.05)$. Although this is partly explained by difference in prevalence of $\mathrm{CaP}$ disease due to a difference in biopsy population (relatively more prior negative men in the center with lower $\mathrm{CaP}$ detection), our results also demonstrate that there is still a need to achieve higher consistency in the reporting of mpMRI and targeting of suspicious lesions. Given the fact that both centers had a comparable amount of missed significant $\mathrm{CaP}$ on $\mathrm{TBx}$ ( $4 \%$ vs. $6 \%$ ), $\mathrm{SBx}$ should not be considered as redundant for the time being and should be performed, just as MRI reading and $\mathrm{TBx}$, by dedicated operators with experience in TRUS. A combination of both $\mathrm{SBx}$ as TBx seems necessary in the detection of significant $\mathrm{CaP}$ as both techniques detect non-negligible significant $\mathrm{CaP}$ missed in a stand-alone approach.

\section{Conclusions}

In men with a prebiopsy positive mpMRI, TBx detects high-GS $\mathrm{CaP}$ while reducing insignificant $\mathrm{CaP}$ detection as compared to SBx. SBx and TBx as stand-alone missed significant $\mathrm{CaP}$ in $13 \%$ and $10 \%$ of the men with significant $\mathrm{CaP}$ on biopsy, respectively. A combination of $\mathrm{SBx}$ and TBx remains necessary for the most accurate assessment of detection, grading and localization of $\mathrm{CaP}$.

\section{Author disclosure statement}

All authors declare no conflicts of interests.

\section{Acknowledgments}

None.

\section{Ethical considerations}

The medical ethics review committee of each participating center granted a waiver for the collection and analysis of the clinical data in a prospective database. The trial was conducted in accordance to the Good Clinical Practice.

\section{Supplementary materials}

Supplementary material associated with this article can be found in the online version at https://doi:10.1016/j.uro lonc.2019.01.005.

\section{References}

[1] Schoots IG, Roobol MJ, Nieboer D, Bangma CH, Steyerberg EW, Hunink MGM. Magnetic resonance imaging-targeted biopsy may enhance the diagnostic accuracy of significant prostate cancer detection compared to standard transrectal ultrasound-guided biopsy: a systematic review and meta-analysis. Eur Urol 2015;68(3):438-50. https://doi.org/10.1016/j.eururo.2014.11.037.

[2] Kasivisvanathan V, Rannikko AS, Borghi M, et al. MRI-targeted or standard biopsy for prostate-cancer diagnosis. N Engl J Med 2018. https://doi.org/10.1056/NEJMoa1801993:NEJMoa1801993.

[3] Borkowetz A, Platzek I, Toma M, et al. Direct comparison of multiparametric magnetic resonance imaging (MRI) results with final histopathology in patients with proven prostate cancer in MRI/ ultrasonography-fusion biopsy. BJU Int 2016;118(2):213-20. https:// doi.org/10.1111/bju.13461.

[4] Le JD, Stephenson S, Brugger M, et al. Magnetic resonance imaging-ultrasound fusion biopsy for prediction of final prostate pathology. J Urol 2014;192(5):1367-73. https://doi.org/10.1016/j. juro.2014.04.094.

[5] Lanz C, Cornud F, Beuvon F, et al. Gleason score determination with transrectal ultrasound-magnetic resonance imaging fusion guided prostate biopsies - are we gaining in accuracy? J Urol 2016;195 (1):88-93. https://doi.org/10.1016/j.juro.2015.07.021.

[6] Ahmed HU, El-Shater Bosaily A, Brown LC, et al. Diagnostic accuracy of multi-parametric MRI and TRUS biopsy in prostate cancer (PROMIS): a paired validating confirmatory study. Lancet 2017;389 (10071):815-22. https://doi.org/10.1016/S0140-6736(16)32401-1.

[7] Simmons L, Kanthabalan A, Arya M, et al. The PICTURE study: diagnostic accuracy of multiparametric MRI in men requiring a repeat prostate biopsy. Br J Cancer 2017;116(9):1159-65.

[8] Mottet N, Bellmunt J, Bolla M, et al. EAU-ESTRO-SIOG guidelines on prostate cancer. Part 1: screening, diagnosis, and local treatment with curative intent. Eur Urol 2017;71(4):618-29. https://doi.org/ 10.1016/j.eururo.2016.08.003.

[9] Siddiqui MM, Rais-Bahrami S, Turkbey B, et al. Comparison of MR/ ultrasound fusion-guided biopsy with ultrasound-guided biopsy for the diagnosis of prostate cancer. JAMA J Am Med Assoc 2015;313 (4):390-7. https://doi.org/10.1001/jama.2014.17942.

[10] Moldovan PC, Van den Broeck T, Sylvester R, et al. What is the negative predictive value of multiparametric magnetic resonance imaging in excluding prostate cancer at biopsy? a systematic review and meta-analysis from the european association of urology prostate cancer guidelines panel. Eur Urol 2017;72(2):250-66. https://doi.org/ 10.1016/j.eururo.2017.02.026.

[11] Moore CM, Robertson NL, Arsanious N, et al. Image-guided prostate biopsy using magnetic resonance imaging-derived targets : a systematic review. 2013;63:125-140.

[12] Fütterer JJ, Briganti A, De Visschere P, et al. Can clinically significant prostate cancer be detected with multiparametric magnetic resonance imaging? a systematic review of the literature. Eur Urol 2015;68(6):1045-53. https://doi.org/10.1016/j.eururo.2015.01.013.

[13] Schouten MG, van der Leest M, Pokorny M, et al. Why and where do we miss significant prostate cancer with multi-parametric magnetic resonance imaging followed by magnetic resonance-guided and 
transrectal ultrasound-guided biopsy in biopsy-naïve men? Eur Urol. 2017:6-13. doi:10.1016/j.eururo.2016.12.006.

[14] Filson CP, Natarajan S, Margolis DJA, et al. Prostate cancer detection with magnetic resonance-ultrasound fusion biopsy: the role of systematic and targeted biopsies. Cancer 2016;122(6):884-92. https:// doi.org/10.1002/cncr.29874.

[15] Fulgham PF, Rukstalis DB, Turkbey IB, et al. AUA policy statement on the use of multiparametric magnetic resonance imaging in the diagnosis, staging and management of prostate cancer. J Urol 2017;198(4):832-8. https://doi.org/10.1016/j.juro.2017.04.101.

[16] Moore CM, Kasivisvanathan V, Eggener S, et al. Standards of reporting for MRI-targeted biopsy studies (START) of the prostate: Recommendations from an international working group. Eur Urol 2013;64 (4):544-52. https://doi.org/10.1016/j.eururo.2013.03.030.

[17] Weinreb JC, Barentsz JO, Choyke PL, et al. PI-RADS prostate imaging-reporting and data system: 2015, Version 2. Eur Urol 2016;69 (1):16-40. https://doi.org/10.1016/j.eururo.2015.08.05.

[18] Natarajan S, Marks LS, Margolis DJA, et al. Clinical application of a 3D ultrasound-guided prostate biopsy system. Urol Oncol Semin Orig Investig 2011;29(3):334-42. https://doi.org/10.1016/j. urolonc.2011.02.014.

[19] Gayet M, van der Aa A, Schmitz P, et al. 3D Navigo ${ }^{\text {TM }}$ versus TRUSguided prostate biopsy in prostate cancer detection. World J Urol 2016;34(9):1255-60. https://doi.org/10.1007/s00345-016-1775-9.

[20] Epstein JI, Egevad LL, Amin MB, et al. The 2014 International Society of Urological Pathology (ISUP) consensus conference on gleason grading of prostatic carcinoma: definition of grading patterns and proposal for a new grading system. Am J Surg Pathol 2016;40(2):244-52.

[21] Panebianco V, Barchetti F, Sciarra A, et al. Multiparametric magnetic resonance imaging vs. standard care in men being evaluated for prostate cancer: A randomized study. Urol Oncol Semin Orig Investig 2015;33(1):17.e1-7. https://doi.org/10.1016/j.urolonc.2014.09.013.

[22] Radtke JP, Schwab C, Wolf MB, et al. Multiparametric Magnetic Resonance Imaging (MRI) and MRI-transrectal ultrasound fusion biopsy for index tumor detection: correlation with radical prostatectomy specimen. Eur Urol 2016;70(5):846-53. https://doi.org/ 10.1016/j.eururo.2015.12.052.

[23] Wegelin O, Henken K, Somford D, et al. An ex vivo phantom validation study of an MRI-transrectal ultrasound fusion device for targeted prostate biopsy. J Endourol 2016;30(6):685-91. https://doi.org/ 10.1089/end.2015.0864.

[24] Westhoff N, Siegel FP, Hausmann D, et al. Precision of MRI/ ultrasound-fusion biopsy in prostate cancer diagnosis: an ex vivo comparison of alternative biopsy techniques on prostate phantoms. World J Urol 2017;35(7):1015-22. https://doi.org/10.1007/ s00345-016-1967-3.

[25] Wegelin O, van Melick HHE, Hooft L, et al. Comparing three different techniques for magnetic resonance imaging-targeted prostate biopsies: a systematic review of in-bore versus magnetic resonance imaging-transrectal ultrasound fusion versus cognitive registration. is there a preferred technique? Eur Urol 2017;71(4):517-31. https://doi. org/10.1016/j.eururo.2016.07.041.

[26] Russo F, Regge D, Armando E, et al. Detection of prostate cancer index lesions with multiparametric magnetic resonance imaging (mp-
MRI) using whole-mount histological sections as the reference standard. BJU Int 2016;118(1):84-94. https://doi.org/10.1111/bju.13234.

[27] Porpiglia F, De Luca S, Checcucci E, et al. Comparing Image-guided targeted Biopsies to Radical Prostatectomy Specimens for Accurate Characterization of the Index Tumor in Prostate Cancer. Anticancer Res 2018;38(5):3043-7. https://doi.org/10.21873/anticanres. 12560.

[28] Cooper CS, Eeles R, Wedge DC, et al. Analysis of the genetic phylogeny of multifocal prostate cancer identifies multiple independent clonal expansions in neoplastic and morphologically normal prostate tissue. Nat Genet 2015;47(4):367-72. https://doi.org/ 10.1038/ng.3221.

[29] Le JD, Tan N, Shkolyar E, et al. Multifocality and prostate cancer detection by multiparametric magnetic resonance imaging: Correlation with whole-mount histopathology. Eur Urol 2015;67 (3):569-76. https://doi.org/10.1016/j.eururo.2014.08.079.

[30] Scheltema MJ, Tay KJ, Postema AW, et al. Utilization of multiparametric prostate magnetic resonance imaging in clinical practice and focal therapy: report from a Delphi consensus project. World J Urol 2017;35(5):695-701. https://doi.org/10.1007/s00345-016-1932-1.

[31] van Hove A, Savoie PH, Maurin C, et al. Comparison of imageguided targeted biopsies versus systematic randomized biopsies in the detection of prostate cancer: a systematic literature review of well-designed studies. World J Urol 2014;32(4):847-58. https://doi. org/10.1007/s00345-014-1332-3.

[32] Rouvière O, Puech P, Renard-Penna R, et al. Use of prostate systematic and targeted biopsy on the basis of multiparametric MRI in biopsy-naive patients (MRI-FIRST): a prospective, multicentre, paired diagnostic study. Lancet Oncol 2018;2045(18):1-10. https:// doi.org/10.1016/S1470-2045(18)30569-2.

[33] van der Leest M, Cornel E, Isra€el B, et al. Head-to-head comparison of transrectal ultrasound-guided prostate biopsy versus multiparametric prostate resonance imaging with subsequent magnetic resonanceguided biopsy in biopsy-na€ıve men with elevated prostate-specific antigen: a large prospective multicenter clinical study. Eur Urol 2018. pii: S03022838(18)30880-7. https://doi.org/10.1016/j.eururo.2018.11.023. [Epub ahead of print].

[34] Abraham NE, Mendhiratta N, Taneja SS. Patterns of repeat prostate biopsy in contemporary clinical practice. J Urol 2015;193(4):117884. https://doi.org/10.1016/j.juro.2014.10.084.

[35] Sing H, Canto EI, Shariat SF, et al. Predictors of prostate cancer after initial negative systematic 12 core biopsy. J Urol 2004;171(5):1850-4.

[36] Di Campli E, Delli Pizzi A, Seccia B, et al. Diagnostic accuracy of biparametric vs multiparametric MRI in clinically significant prostate cancer: comparison between readers with different experience. Eur J Radiol 2018;101:17-23. https://doi.org/10.1016/j.ejrad.2018.01.028.

[37] De Visschere P, Lumen N, Ost P, Decaestecker K, Pattyn E, Villeirs G. Dynamic contrast-enhanced imaging has limited added value over T2-weighted imaging and diffusion-weighted imaging when using PI-RADSv2 for diagnosis of clinically significant prostate cancer in patients with elevated PSA. Clin Radiol 2017;72(1):23-32. https:// doi.org/10.1016/j.crad.2016.09.011.

[38] Kweldam CF, Kümmerlin IP, Nieboer D, et al. Prostate cancer outcomes of men with biopsy Gleason score 6 and 7 without cribriform or intraductal carcinoma. Eur J Cancer 2016;66:26-33. https://doi. org/10.1016/j.ejca.2016.07.012. 